\title{
Original Research \\ An animal study on the effect of topically administered ambroxol for dry eye
}

\author{
Zixuan Wang ${ }^{1}$, Li Yu ${ }^{1, *}$, Shaochong Zhang ${ }^{1}$, Zhenhan Wang ${ }^{2}$, Mingwu Wang ${ }^{3,4}$ \\ ${ }^{1}$ Shenzhen Eye Hospital, School of Optometry, Shenzhen University, 518040 Shenzhen, Guangdong, China \\ ${ }^{2}$ Shenzhen Tusang Medical Science Corp, 518000 Shenzhen, Guangdong, China \\ ${ }^{3}$ Department of Ophthalmology and Vision Science, University of Arizona College of Medicine, Tucson, AZ 85710, USA \\ ${ }^{4}$ NeuVision Medical Institute, Tucson, AZ 85718, USA \\ *Correspondence: jasmine791117@163.com (Li Yu) \\ Academic Editor: Shikun He \\ Submitted: 19 October 2021 Revised: 18 November 2021 Accepted: 23 November 2021 Published: 5 January 2022
}

\begin{abstract}
Objective: To evaluate the effect of $0.2 \%$ ambroxol eye drop on tear secretion and corneal healing on a rabbit dry eye model, and to delineate potential underlying mechanisms. Materials and method: A mixed mechanism dry eye model was created using 12 healthy New Zealand rabbits by excision of the main lacrimal glands, Harderian gland and nictitating membrane. Establishment of the model was confirmed by the decrease of Schirmer I and increase of corneal fluorescein staining scores. Two weeks after model creation, the rabbits were randomly and evenly divided into $\mathrm{NaCl}, 0.1 \%$ sodium hyaluronate and $0.2 \%$ ambroxol groups. Each group was administered the respective eye drops 4 times a day for four weeks. The Schirmer I test and corneal fluorescein staining were performed at two and four weeks. After four weeks of treatment, the animals were sacrificed and the conjunctiva and eyelid specimens collected. Inflammatory factors IL-8, TNF- $\alpha$, and goblet cell specific mucin MUC5AC were measured by ELISA while the lid meibomian gland was evaluated by oil red O staining. Results: Compared with the baseline, 2 weeks after the surgery, Schirmer I test value decreased significantly (20.35 $\pm 5.18 \mathrm{~mm} / 5 \mathrm{~min}$ vs $13.95 \pm 4.64 \mathrm{~mm} / 5 \mathrm{~min}, p<0.01)$, and the fluorescein staining score increased significantly $(0.5 \pm 0.6 \mathrm{vs} 5.5 \pm$ $1.4, p<0.01)$. After four weeks of treatment, compared with the $\mathrm{NaCl}$ and sodium hyaluronate groups, tear secretion in ambroxol group increased significantly $(p<0.01)$, while the corneal fluorescence staining score decreased significantly $(p<0.01)$. In the conjunctival tissue, significant decrease was seen in TNF- $\alpha(p<0.01)$ and IL-8 [ $p$ (unilateral) $<0.05$ ] concentrations in ambroxol group, and significant increase in MUC5AC concentration $(p<0.01)$ in ambroxol group as well. The lipid content in the lid meibomian glands appeared increased after the administration of ambroxol. Conclusion: The present rabbit dry eye model study demonstrated potentials of topically administered $0.2 \%$ ambroxol in stimulating tear and mucin secretion, inhibiting ocular surface inflammation, promoting corneal healing, and possibly augmenting meibomian gland lipid production.
\end{abstract}

Keywords: Ambroxol; Secretagogue; Dry eye disease; Rabbit dry eye model; Inflammatory cytokines; MUC5AC; ICAM-1; Lifitegrast

\section{Introduction}

Altered tear film (TF) composition is a common cause of dry eye disease (DED) which can further lead to ocular surface inflammation and loss of tear homeostasis. There are two types of DED in current classification - aqueousdeficient and evaporative, related directly to decrease in aqueous or lipid components of TF [1]. Evaporative DED is more common than aqueous-deficient type, but more than one-third of patients will have a combination of both. DED represents one of the biggest unmet needs in ophthalmology. Current methods can often alleviate but not cure DED. Therefore, in-depth study of the pathogenesis of DED, as well as the discovery of new drugs are still vitally important.

The natural hydrophobicity of cell membrane lipids requires mucin to fix the tear film on the ocular surface for lubrication and protection. Our early study found that transconjunctival fluid transport driven by osmotic pressure gradient is mainly carried out through aquaporin, and its in- creased expression can drive the expression and secretion of MUC5AC, the most important gel-forming mucin in tears, from conjunctival epithelial goblet cells [2]. We have also recently discovered that ambroxol can stimulate the expression of aquaporin 5 (AQP5) and mucin 5AC (MUC5AC) in vitro, and can promote tear secretion in normal rabbits [3], demonstrating potential as a drug for DED. Ambroxol is a secretolytic and mucoactive agent primarily used to treat respiratory diseases associated with viscid mucus [4]. Ambroxol is reported as having multiple clinically relevant actions including reduction of mucus viscosity, stimulation of airway surfactant secretion [5], anti-inflammation [6] and analgesia [7]. All these pharmaceutical properties are of potential benefit for DED.

To date, various lacrimal deficiency dry eye animal models have been employed in studies such as by inducing autoimmune reaction, removing lacrimal gland, blocking nerve, and using parasympathetic nerve inhibitor [8-10], all with pros and cons. In this study, we chose a mixed- 
mechanism rabbit dry eye model reliably used by our group [11], and used $\mathrm{NaCl}$ and sodium hyaluronate eye drops as controls, to evaluate the effect of $0.2 \%$ ambroxol eye drop on dry eye. We also attempted to explore other potential mechanisms of action by ambroxol in this dry eye model.

\section{Materials and method}

\subsection{Experimental animals and ethics statement}

A total of 12 male New Zealand white rabbits (24 eyes, Xinhua Research Animal Farm, Guangzhou, Guangdong, China) weighing 2.0-2.5 kg were purchased. All animals had passed quarantine inspection and been given one week adaptation time to the new environment prior to enrollment into the study. Corneal fluorescein staining score was recorded and Schirmer I test conducted in each eye as baseline prior to surgery. The animals were reared at the laboratory of Shenzhen Lingxian Medical Service Co., Ltd., under standard conditions $\left(22 \pm 2{ }^{\circ} \mathrm{C}, 40 \% \pm 5 \%\right.$ relative humidity, and a 12-hour light-dark cycle) with free access to food and water throughout the experiment. The surgeries and study were also carried out at the same facility. The study was conducted in compliance with the Tenets of the Declaration of Helsinki and ARVO statement for the use of animals in ophthalmic and visual research. The protocol was approved by Shenzhen Eye Hospital Institutional Animal Care and Use Committees. All animal tests were performed by designated animal facility staff trained by and following the instructions of Drs. Mingwu Wang and Li Yu for purpose of examiner-masking. All surgeries were performed by Dr. Li Yu.

\subsection{Establishment of dry eye model}

The main lacrimal gland, Harderian gland (HG) and nictitating membrane (NM) were excised in both eyes as per previous publications $[12,13]$ with minor modification. Identical procedure was performed on both eyes. Briefly, under general anesthesia, the skin of both periocular regions was shaved, disinfected with $5 \%$ betadine and draped. The orbital and palpebral lobes of the superior lacrimal gland was first removed (Fig. 1a,b,c,f) as described previously [13]. Subsequently, the inferior lobe was removed through a linear skin incision along the inferior and lateral orbital rims (Fig. 1d,f). The NM was then snipped off at its base, the HG extracted through the same wound and removed in its entirety (Fig. 1e,f). Hemostasis was achieved by gentle tamponade. The orbit septum and skin wound were then closed separately with interrupted 5-0 vicryl sutures. Ofloxacin ophthalmic ointment (Santen Pharmaceutical Co., Ltd., Osaka, Japan) was administered topically at the surgical wounds twice a day for 7 days for infection prophylaxis.

All animals were evaluated prior to model creation surgeries and 2 weeks after for confirmation of the establishement of the dry eye medol. After 2 weeks, the 12 animals were randomly divided into 3 groups: $0.9 \% \mathrm{NaCl}$,
$0.1 \%$ sodium hyaluronate (Lianbang Pharmatheutical Ltd, Corp, Zhuhai, China, Lot\#: M0861201-N02) and 0.2\% ambroxol (MedChem Express, Shanghai, USA) groups. Each group had 4 rabbits ( 8 eyes). Each eye then received the respective eye drop 4 times a day, starting from week 2 , and was evaluated every 2 weeks for additional of 4 weeks.

\subsection{Evaluation of animals}

\subsubsection{Corneal fluorescein staining}

The fluorescein sodium staining test paper was first wetted with one drop of normal saline and then the tip of paper gently touched the lower conjunctival sac of each rabbit eye. After several natural blinking, each rabbit eye was observed under the slit lamp with diffuse cobalt blue light and the staining of cornea recorded by photographs. Using the standard NEI15 method, the cornea was divided into five zones: central, upper, lower, temporal and nasal. Each zone was scored according to severity and pattern of staining: 0 for no staining, 1 for scattered punctate staining, 2 for diffuse punctate staining, 3 for filamentous, blot or patchy staining, with a total possible score of 15 points in each eye.

\subsubsection{Schirmer I detection}

A standard $5 \times 35 \mathrm{~mm}$ tear secretion test paper strip was first folded at $5 \mathrm{~mm}$ from its end, and then placed at the lateral one third of the inferior conjunctival sac with no topical anesthesia. After five minutes, the paper strip was taken out, the length of the wet portion from the folded line was recorded. The Schirmer test was repeated three times on the same day and the average value taken for analysis.

\subsubsection{Specimen collection and fixation}

At the end of the study, the animals were sacrificed by air embolism, and the bulbar conjunctival tissue near the superior fornix and a segment of full-thickness superior lid tissue were removed from each rabbit eye. The bulbar conjunctival tissue specimens were blotted with filter paper first and weighed. PBS was then added to the tissue at a 9:1 ratio (e.g., $9 \mathrm{~mL}$ PBS to $1 \mathrm{~g}$ specimen). The conjunctival specimens were homogenized in PBS and placed at $-20{ }^{\circ} \mathrm{C}$ overnight. After repeated freezing and thawing to further break the cell membrane, the tissue homogenates were centrifuged at $5000 \mathrm{rpm}$ for 15 minutes and the supernatants taken for ELISA. The lid tissues containing meibomian glands were fixed in $10 \%$ formalin for 24 hours, then processed for standard histological sectioning.

\subsubsection{Oil red O stain}

Rabbit upper lid tissue sections were deparaffinized and rehydrated first, washed with double distilled water, and then stained with oil red working solution for 10 15 minutes according to manufacture recommended protocol (Shanghai Yuanye Bio-Technology Co., Ltd., Shanghai, China). Last, the sections were counter-stained with hematoxylin for observation under light microscope. The oil content in the tissue appeared bright red, and the cell nucleus blue. 


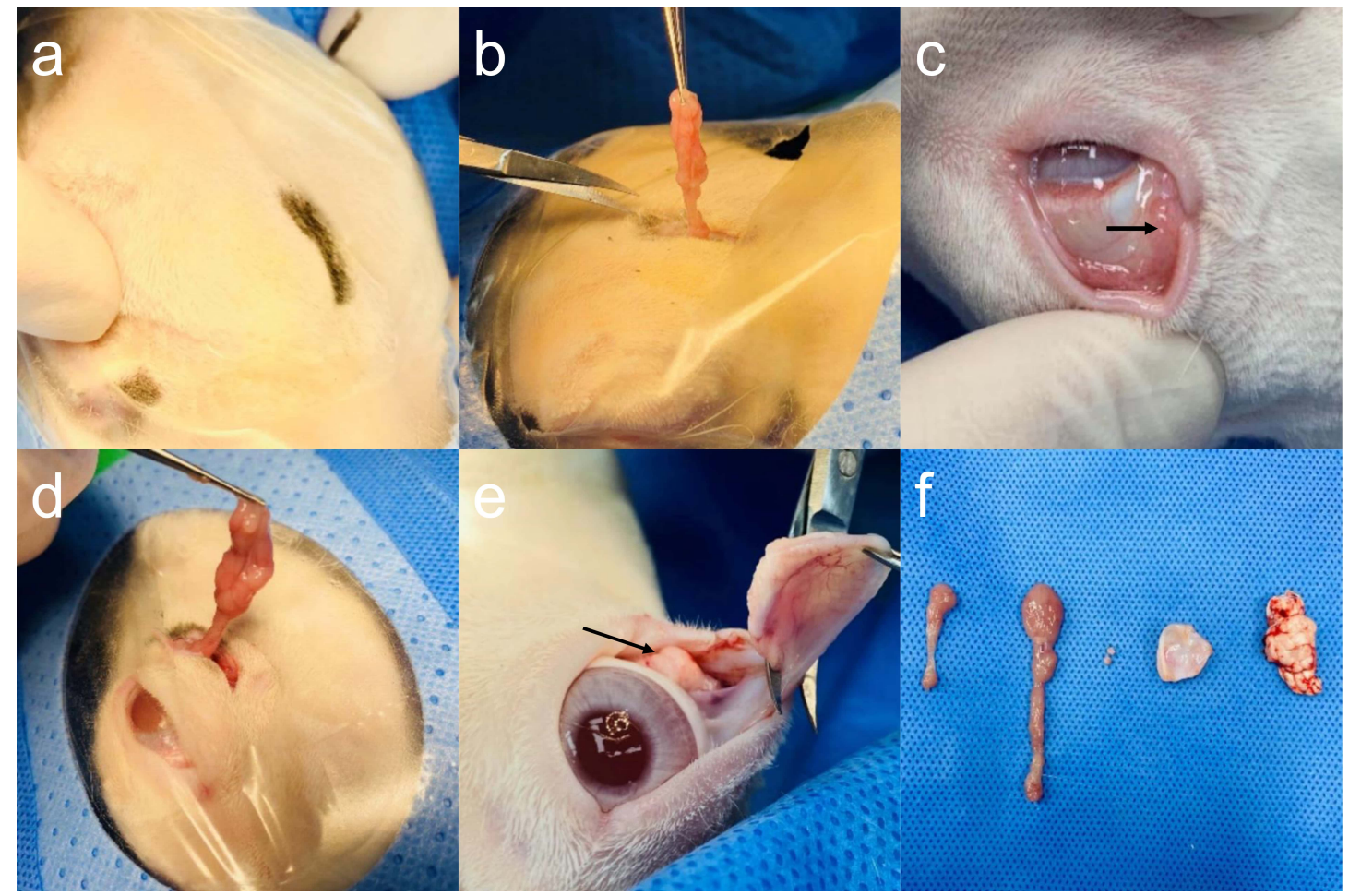

Fig. 1. Surgical steps involved in the creation of a mixed-mechanism rabbit dry eye model. (a) Superior orbital incision. (b) Extraction of the superaorbital lacrimal gland. (c) Identification of the palpebral lacrimal gland for excision. (d) Extraction of the infraorbital lacrimal gland. (e) Excision of the nictitating membrane and Harderian gland. (f) Tissues removed (from left to right) are the supraorbital lacrimal gland, infraorbital lacrimal gland, palpebral lacrimal gland, nictitating membrane, and Harderian gland.

\subsubsection{Assessment of cytokines and goblet cell-specific} mucin

After centrifugation, the supernatants of bulbar conjunctival homogenates were taken for quantitative assessment of IL-8, TNF- $\alpha$ and MUC5AC by ELISA. The standard sample and series dilution solutions were used as positive and assay buffer blank as negative controls. The assay was carried out following the manufacturer's standard protocol (Shanghai Huding Biotechnology Co., Ltd., Shanghai, China). The concentrations of IL- 8 , TNF- $\alpha$ and mucin MUC5AC were calculated by Curve Expert 1.4 software (Hyams Development, Huntsville, Alabama, USA).

\subsection{Statistical analysis of the data}

GraphPad Prism 8.3.0 software (GraphPad Software, LLC. San Diego, CA, USA) was used for statistical analysis. Statistical significances among Schirmer I values, corneal fluorescein staining scores, cytokine and MUC5AC concentrations before and after ambroxol treatment were first analyzed by one-way Anova, and in each group, further compared by Fishers LSD test.

\section{Results}

\subsection{Establishment of mixed-mechanism rabbit dry eye model}

All 12 rabbits suffered no complications from surgeries and appeared well physically throughout the study. Compared with the baseline, the values of Schirmer I at two weeks after model creation had decreased significantly $(20.35 \pm 5.18 \mathrm{~mm}$ vs $13.95 \pm 4.64 \mathrm{~mm}, p<0.01)$ (Fig. 2), and the corneal fluorescein staining score significantly increased $(0.5 \pm 0.6$ vs $5.5 \pm 1.4, p<0.01)$ (Figs. 3,4). Before the operation, the corneas of rabbits had hardly any staining with fluorescein. Two weeks after the operation, most of the corneas showed various degrees of diffuse punctate to pachy staining (Fig. 3). The decreased tear secretion by about $30 \%$ and increased corneal staining on the epithelium confirmed the establishment of the rabbit dry eye model.

\subsection{Tear secretion increased significantly four weeks after treatment with ambroxol}

There was significant difference in tear secretion at different time in ambroxol administration group $(\mathrm{F}=6.34$, $p<0.01)$ but not in the other two groups. Compared to two weeks after the surgeries, statistically significant increase in tear secretion in the ambroxol group was seen at four weeks after treatment $(p<0.01)$. Four weeks after treat- 


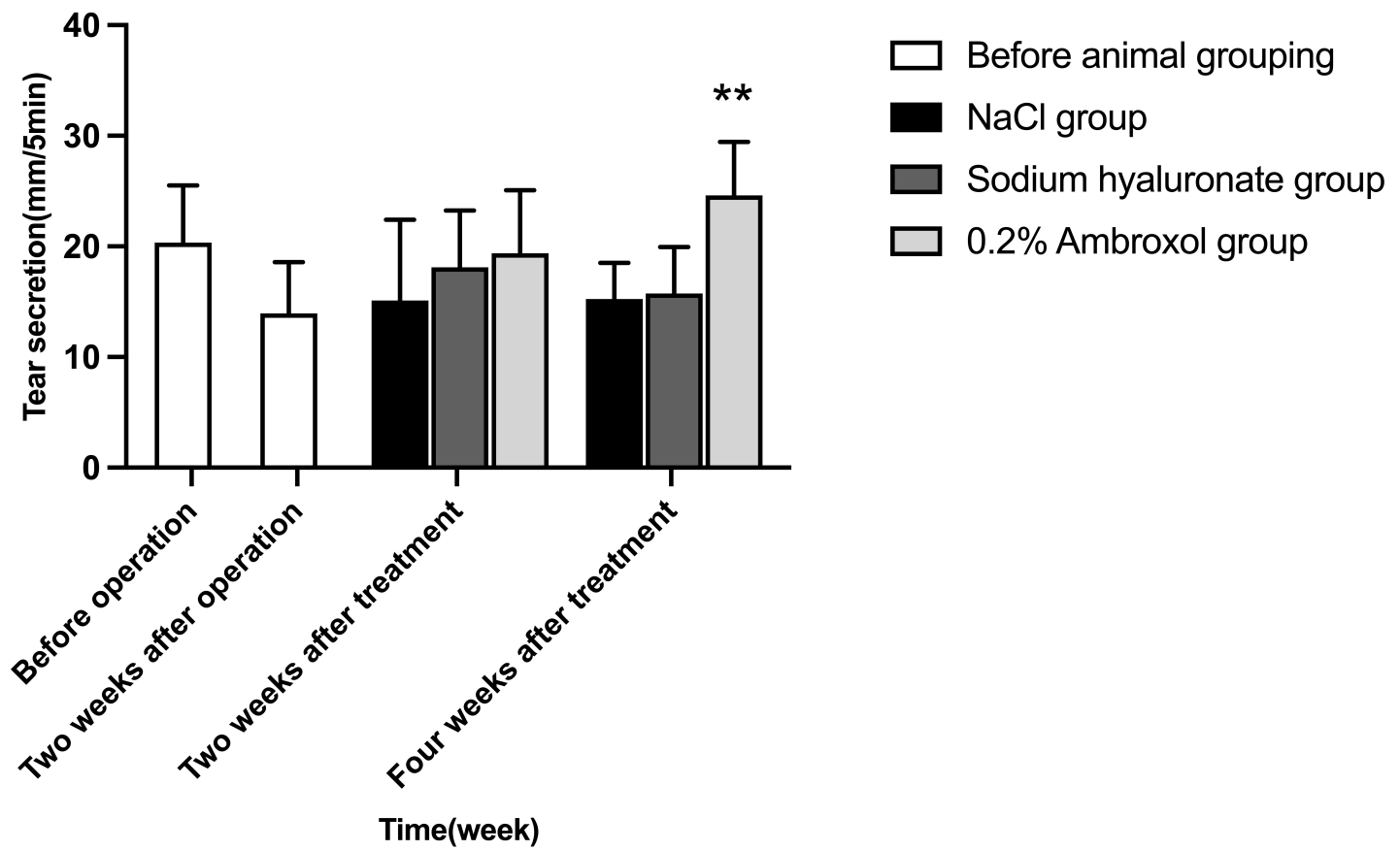

Fig. 2. Comparison of tear secretion among groups at two weeks after operation, two and four weeks after treatment. Comparison of ambroxol group with $\mathrm{NaCl}$ group, ${ }^{* *} p<0.01$; Comparison with sodium hyaluronate group, ${ }^{* *} p<0.01$; Comparison with two weeks after the operation, $* * p<0.01$ (univariate analysis of variance, LSD- $t$ test).

ment, there was significant increase in tear secretion among the groups $(\mathrm{F}=12.95, p<0.01)$. Compared with the $\mathrm{NaCl}$ and sodium hyaluronate groups, the tear secretion in the ambroxol group was significantly increased four weeks after treatment ( $p<0.01)$ (Fig. 2).

\subsection{The effect of $0.2 \%$ ambroxol eye drops on corneal injury}

Compared to two weeks after surgeries, two weeks after treatment, the corneal patchy fluorescent staining disappeared in ambroxol group, leaving only focal punctate staining. After two weeks of treatment, sodium hyaluronate group showed scattered punctate staining, whereas $\mathrm{NaCl}$ group showed filamentous staining. Four weeks after treatment, the fluorescent staining of corneas in ambroxol and sodium hyaluronate groups almost disappeared, whereas that in $\mathrm{NaCl}$ group still showed scattered punctate staining (Fig. 3).

Compared to two weeks after surgeries, the corneal fluorescein staining scores in ambroxol and sodium hyaluronate groups were significantly reduced at two and four weeks after the treatment $(p<0.01)$, and there was no significant change in $\mathrm{NaCl}$ group after treatment. Four weeks after treatment, the scores of corneal fluorescein staining in ambroxol group was significantly lower than that in $\mathrm{NaCl}$ group $(p<0.01)$, but there was no difference between ambroxol and sodium hyaluronate groups (Figs. 3,4).
3.4 The effect of $0.2 \%$ Ambroxol eye drops on the expression of inflammatory cytokines and MUC5AC mucin in conjunctival tissue

After four weeks of treatment with respective eye drops, the conjunctival specimens were assessed by ELISA for level of TNF- $\alpha$, IL-8 and MUC5AC, and compared among three groups. The overall comparison of TNF- $\alpha$ concentrations among the three groups was statistically significant $(\mathrm{F}=7.08, p<0.01)$. The concentration of TNF$\alpha$ in ambroxol group was significantly lower than that of the other two groups $(p<0.01)$. Compared with the $\mathrm{NaCl}$ and the sodium hyaluronate groups, the level of IL- 8 in ambroxol group was reduced as well ( $p$ (unilateral) $<0.05$ ) The conjunctival MUC5AC concentration was significantly different among groups after four weeks of treatment $(\mathrm{F}=$ 13.10, $p<0.01$ ). Compared with the $\mathrm{NaCl}$ and the sodium hyaluronate groups, MUC5AC in ambroxol group was significantly increased $(p<0.01)$ (Table 1$)$.

3.5 Lipid production in the meibomian glands appeared to have increased after treatment with ambroxol

The results of oil red $\mathrm{O}$ staining of meibomian glands showed that after four weeks of treatment, compared with $\mathrm{NaCl}$ and sodium hyaluronate groups, there appeared to have more bright red oil content in the ambroxol group (Fig. 5). 


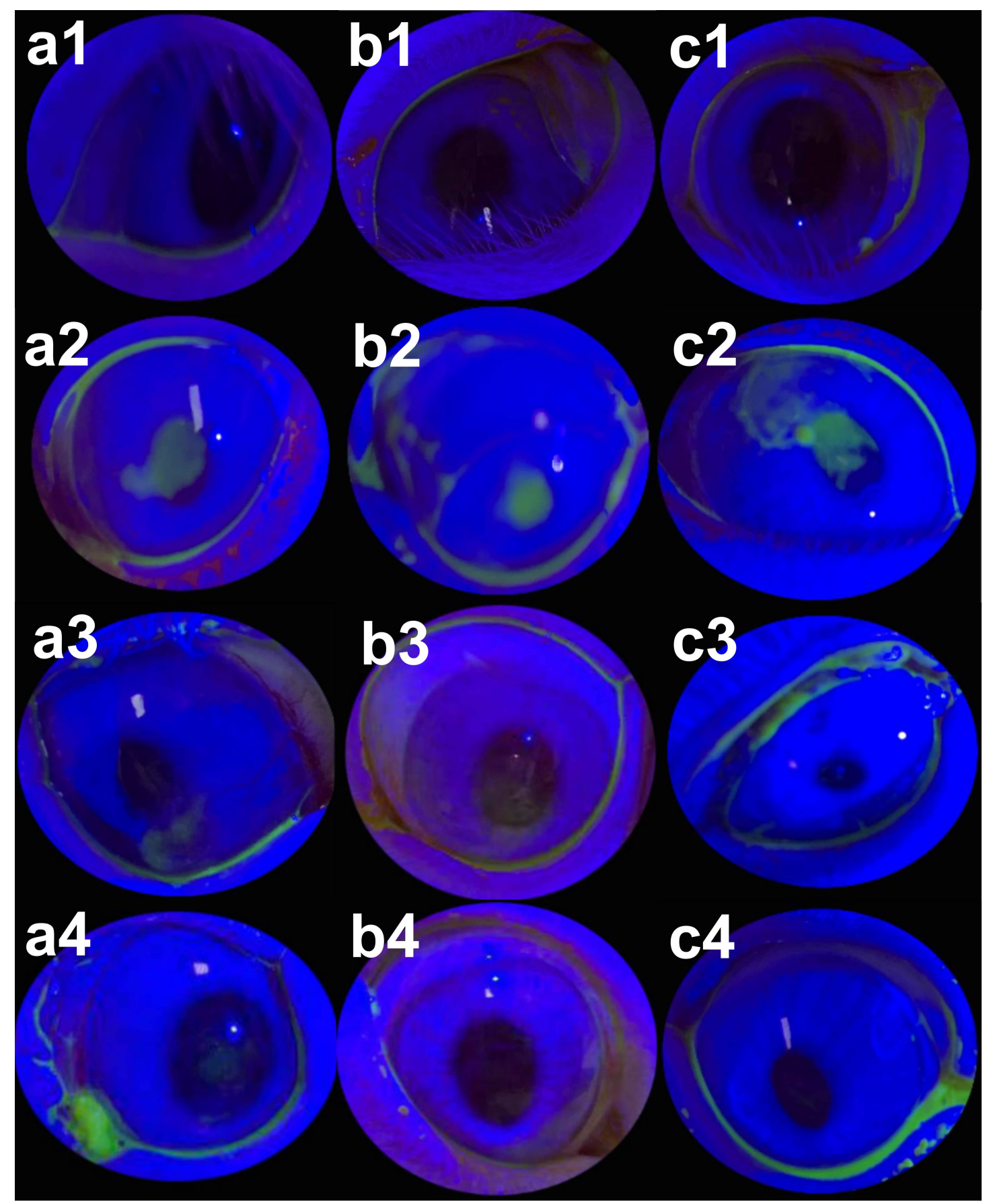

Fig. 3. Results of corneal fluorescein staining before operation (1), two weeks after operation (2), two (3) and four weeks (4) after treatment with NaCl. (a) Sodium hyaluronate (b) and $0.2 \%$ ambroxol (c). No obvious staining of fluorescein staining noted in rabbit corneas before operation $(\mathrm{a} 1, \mathrm{~b} 1, \mathrm{c} 1)$. Moderate degrees of patchy epithelial defects seen in the corneas two weeks after operation (a2,b2,c2). The rabbit corneas in $\mathrm{NaCl}$ group showed filamentary staining two weeks after treatment (a3) and moderate punctate staining 4 weeks after treatment (a4). The rabbit corneas in sodium hyaluronate group showed scattered punctate staining two weeks after treatment (b3) and minimal fluorescent staining four weeks after treatment (b4). The rabbit corneas in ambroxol group showed localized central punctate staining two weeks after treatment (c3) and hardly any staining four weeks after treatment (c4).

\section{Discussion}

According to the DEWS II 2017 report, dry eye is an ocular surface disease associated with multiple risk factors $[14,15]$. The fundamental characteristic of DED is the dis- ruption of the TF homeostasis, combined with various ocular symptoms. Instability of the TF, increased osmolarity and inflammation, damage to the ocular surface, decreased androgen level, and the neurosensory abnormalities all play important roles in the pathogenesis of DED [15]. Com- 


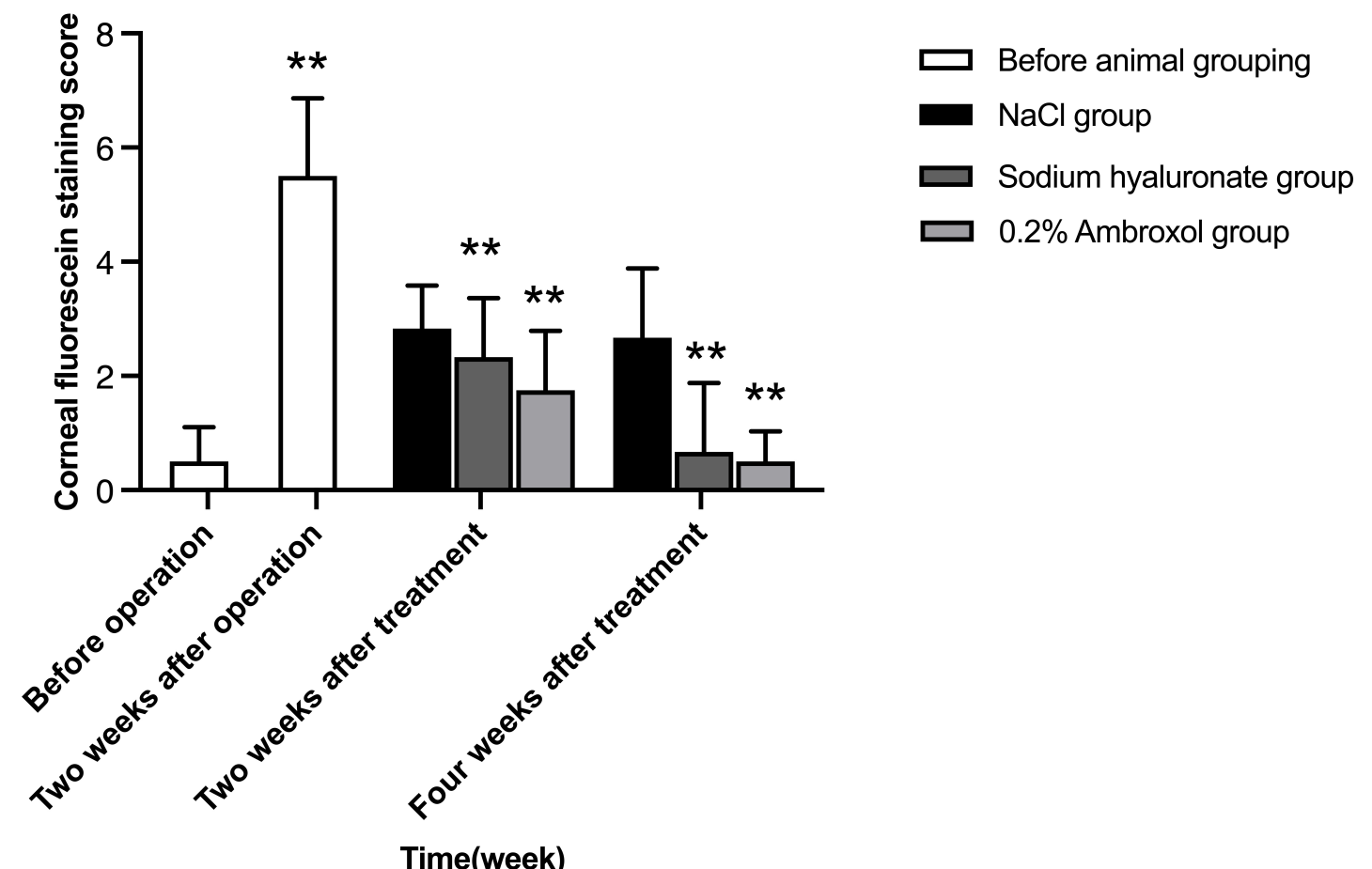

Fig. 4. Comparison of corneal fluorescein staining scores before operation, two weeks after operation, two and four weeks after treatment among groups. $\mathrm{F}$ (group) $=4.62, p<0.01 ; \mathrm{F}($ time $)=92.28, p=0.01$. Comparison with pre-operation two weeks after operation, ${ }^{* *} p<0.01$; Comparison with two weeks after operation two and four weeks after treatment, ${ }^{* *} p<0.01$; Comparison of ambroxol group and sodium hyaluronate group with $\mathrm{NaCl}$ group four weeks after treatment, ${ }^{* *} p<0.01$ (two-way ANOVA, LSD- $t$ test).

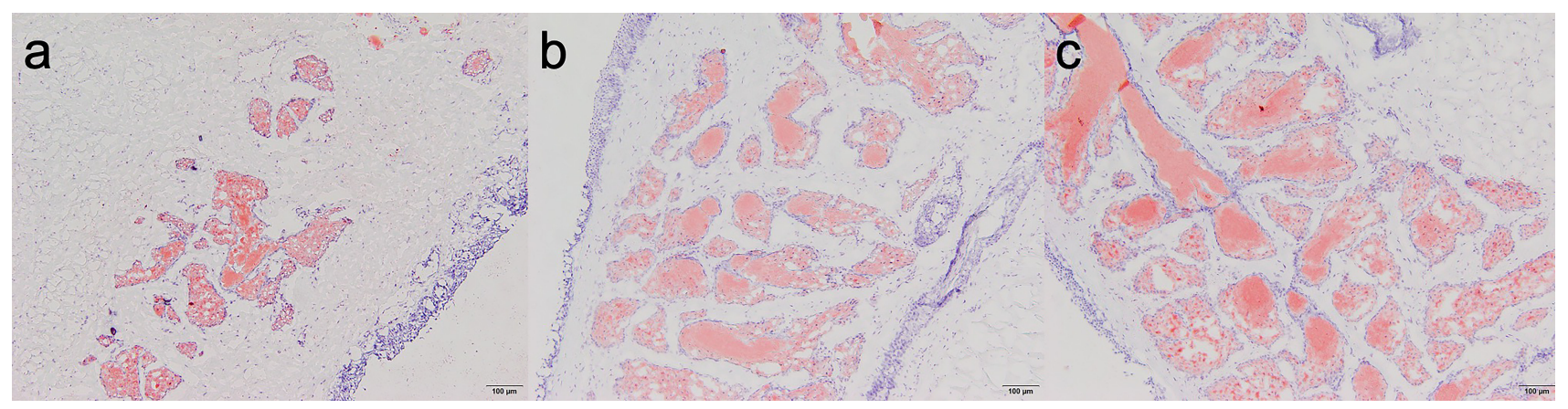

Fig. 5. Oil red O staining of rabbit eyelid meibomian glands among groups four weeks after treatment $(\mathrm{HE} \times 100$, scale $=100$ $\mu \mathbf{m})$. Few bright red oil droplets seen in the $\mathrm{NaCl}$ group (a). Moderate bright red oil droplets seen in the sodium hyaluronate group (b). Rich bright red oil droplets in the $0.2 \%$ ambroxol group seen in comparison with $\mathrm{NaCl}$ and sodium hyaluronate group (c).

pared with rats and mice, the globe size, anatomical structure, physiological function of tissues and the histoarchitectural features of lacrimal glands in rabbits resemble more closely those in human [16]. In addition, rabbit eyes are more suitable to slit lamp microscope observation and photography. Hence, our group commonly use rabbits to create a mixed-mechanism model to study DED. In present study, two weeks after model creation, the Schirmer I test value had significantly decreased from the baseline by about $30 \%$, and the corneal fluorescein staining score significantly increased. The reliability and reproducibility of this model is truly remarkable.
The TF is composed of water, lipid and mucus layers to protect the cornea from exogenous microorganisms, chemical substances and environmental pollutants, and to maintain a smooth refractive surface required for clear vision [17]. Hence, maintaining the homeostasis of the TF is the key in DED treatment.

Our earlier research suggested that augmentation of the AQP function in the conjunctiva has the potential to promote the secretion of tear and MUC5AC, the gel-forming mucin secreted by goblet cells [2,11]. As an active agent on the ocular surface, MUC5AC can help evenly disperse the aqueous layer of the TF [18]. Aqueous and mucin defi- 
Table 1. Comparison of inflammatory cytokines and MUC5AC expression in rabbit conjunctival tissue among groups four weeks after treatment $(x \pm \mathbf{s})$.

\begin{tabular}{lcccr}
\hline Group & Eyes/group & Concentration of TNF- $\alpha(\mathrm{pg} / \mathrm{mL})$ & Concentration of IL-8 $(\mathrm{ng} / \mathrm{L})$ & Concentration of MUC5AC $(\mu \mathrm{g} / \mathrm{L})$ \\
\hline Ambroxol & 8 & $1171.34 \pm 68.84^{* *}$ & $63.89 \pm 2.44^{*}$ & $123.98 \pm 1.81^{* *}$ \\
\hline $\mathrm{NaCl}$ & 8 & $1325.20 \pm 132.58$ & $67.70 \pm 5.06$ & $106.53 \pm 9.42$ \\
\hline Sodium hyaluronate & 8 & $1331.93 \pm 75.07$ & $67.02 \pm 2.84$ & $115.67 \pm 3.55$ \\
\hline F value & & 7.08 & 2.50 & 13.10 \\
\hline$p$ value & & $<0.01$ & 0.11 & $<0.01$ \\
\hline
\end{tabular}

Note: Comparison of ambroxol with $\mathrm{NaCl}$ group and Sodium hyaluronate, ${ }^{*} p<0.05,{ }^{*} p<0.01$ (univariate analysis of variance, LSD- $t$ test).

ciency both contribute to the etiology of DED $[19,20]$. Ambroxol, an active metabolite of bromhexine, is a secretolytic and mucoactive drug primarily used to treat respiratory diseases associated with viscid mucus [4]. In addition to reducing the viscosity of mucus and stimulating the secretion of airway surfactants [5], ambroxol is also known to possess properties of anti-inflammation [6] and analgesia [7]. We have demonstrated recently that ambroxol can stimulate tear and mucin production in normal rabbits by upregulating the expression of AQP and MUC5AC, and hence may have the potential to be a DED drug [3]. The present study further tested the effect of ambroxol on the rabbit dry eye model and shed new light on mode of action by this drug.

In reference to data from previous studies, the present study chose to test $0.2 \%$ ambroxol in a mixed mechanism rabbit dry eye model, in comparison to $\mathrm{NaCl}$ and sodium hyaluronate groups as controls. Four weeks after treatment, $0.2 \%$ ambroxol indeed significantly promoted the secretion of tears on the ocular surface and the recovery of corneal epithelial injury. This study is significant since it is the first time efficacy for DED was demonstrated in vivo. Consistent with previous findings at tissue and cellular levels, ambroxol promoted the production of MUC5AC in the conjunctival tissue as well. Through histological examination of eyelids, the lipid component in the meibomian glands also appeared to have increased, as reflected by oil red $\mathrm{O}$ staining. Bromhexine, the pro-drug of ambroxol, has been shown to increase neutral lipid production in cultured conjunctival cells [21]. Its mechanism may be related to the up-regulation of CHML-mediated Rab7 expression which triggers the change of lysosomal activity and enhances the intracellular transport of many substances such as mucin and lipid droplets [21].

To further explore the mechanism of action, the present study assessed the effect of ambroxol on inhibiting ocular surface tissue inflammation. Four weeks after treatment, the levels of TNF- $\alpha$ and IL- 8 in rabbit conjunctival tissue were significantly lower than that of the control groups. It was not surprising to see decreased TNF- $\alpha$ level since bromhexine was shown to inhibit TNF- $\alpha$ secretion in human conjunctival tissue [21]. The influence seen of ambroxol on IL-8 on the other hand, is tantalizing. IL8 is known to be one of the most potent chemoattractant molecules that, among several other functions, is responsible for guiding neutrophils to sites of injury in tissues [22]. Inhibiting IL-8 signal therefore may reduce further ocular surface tissue injury from neutrophils in DED.

In addition to reducing levels of key inflammatory cytokines such as TNF- $\alpha$ and IL-8, ambroxol's ability to effectively reduce the expression of intercellular adhesion molecule-1 (ICAM-1) in tissues [23] may also contribute to the inhibition of $\mathrm{T}$ cell-related inflammation. On the ocular surface, the interaction between ICAM-1 and lymphocyte function associated antigen-1 (LFA-1) on T cells is the key to incite inflammation in DED [24]. Interaction between the two molecules leads to the recruitment of activated T cells, the activation of newly recruited $\mathrm{T}$ cells and the release of pro-inflammatory cytokines, which is believed to be the main reason for the persistent inflammation in the pathogenesis of DED [15]. Future study is warranted to see if ambroxol can achieve similar anti-inflammatory effect as Lifitegrast [25] by reducing the availability if ICAM-1 and thereby lowering the interaction between ICAM-1 and LFA-1 on conjunctival cells.

Conjunctival epithelium regulates mucosal hydration mainly through the coupling process of sodium ion absorption mediated by cellular sodium channel and chloride ion secretion mediated by cystic fibrosis transmembrane conductance regulator (CFTR). The transconjunctival fluid transport powered by the osmotic pressure gradient is carried out by aquaporins. Evidence shows that ambroxol can up-regulate the expression of CFTR protein and enhance the transmembrane secretion of chloride ions on the surface of respiratory epithelial cells [26]. In addition to promoting the function of AQP molecule, we speculate that ambroxol in the conjunctiva could also stimulate the function of CFTR, thereby increasing the secretion of tears.

Ocular surface tissues, especially the conjunctiva, has sufficient compensatory capacity to generate tears required for tear turnover [27]. DED, especially that of nonautoimmune nature, can often recover spontaneously. In fact, severe recalcitrant DED often shows a significant loss 
of conjunctival goblet cells or end-stage keratosis [17], indicating that the conjunctival tissue is irreversibly damaged and the compensatory capacity seriously impaired. Therefore, early intervention to maintain the normal function of ocular surface tissues should be an important component in DED treatment. Diquafosol sodium and rebamipide, both promote the secretion of tears and mucin in dry eye patients. Diquafoxol sodium has a P2Y2 receptor agonist activity comparable to UTP [28]. Rebamipide is a quinolinone derivative with mucin secretagogue activity [29]. Ambroxol not only promotes the secretion of conjunctival tears, mucins, and even possibly lipids, but also has antiinflammatory property, which are all desirable actions as a DED medicine.

Given that DED is a multifactorial disease which requires a multifaceted approach for management, drugs of multiple pharmaceutical properties obviously have more advantages over single action ones. Ambroxol distinguishes itself from other current single mechnisam drugs on the market or in the development pipeline, and is poised to be the next generation drug candidate for DED.

\section{Conclusions}

Ambroxol has the potentials to become the next generation multi action DED drug candidate. Further research exploration of the pharmaceutical properties of ambroxol is warranted in the future.

\section{Abbreviations}

AQP5, aquaporin 5; ARVO, Association for Research in Vision and Ophthalmology; CFTR, cystic fibrosis transmembrane conductance regulator; DED, dry eye disease; DEWS II, Dry Eye Workshop II; ELISA, enzyme linked immunosorbent assay; $\mathrm{ENaC}$, epithelial $\mathrm{Na}^{+}$Channel; HG, harderian gland; NM, nictitating membrane; IL8, interleukin-8; CHML, metabolism-related gene; ICAM1, intercellular adhesion molecule-1; LFA-1, lymphocyte function associated antigen-1; MUC5AC, mucin 5AC; PBS, phosphate buffered saline; TF, tear film; TNF- $\alpha$, tumor necrosis factor- $\alpha$; UTP, uridine triphosphate.

\section{Author contributions}

LY, MW and SZ were responsible for experimental design, interpretation of study results and drafting of the manuscript for publication. LY, ZW (Zixuan Wang) and ZW (Zhenhan Wang) were responsible for study execution and statistical analyses.

\section{Ethics approval and consent to participate}

The protocol was approved by Shenzhen Eye Hospital Institutional Animal Care and Use Committees. The study was conducted in compliance with the Tenets of the Declaration of Helsinki and ARVO statement for the use of animals in ophthalmic and visual research.

\section{Acknowledgment}

Not applicable.

\section{Funding}

Free Exploration Project of Shenzhen Science and Innovation Commission (JCYJ20170306140638792); International Cooperation Project of Shenzhen Science and Innovation Commission (GJHZ20180929145202083); Medical Prevention Combined Ophthalmology Project. Supported by Shenzhen Fund for Guangdong Provincial Highlevel Clinical Key Specialties (No. SZGSP014).

\section{Conflict of interest}

Mingwu Wang holds international patents in the US, Canada, Australia, Korea, Japan and India, for topical application of ambroxol for treatment of ocular surface diseases. The other authors have no commercial or proprietary interest in any concept or products described in this article.

\section{References}

[1] Craig JP, Nichols KK, Akpek EK, Caffery B, Dua HS, Joo C, et al. TFOS DEWS II Definition and Classification Report. The Ocular Surface. 2017; 15: 276-283.

[2] Bhattacharya D, Yu L, Wang M. Expression patterns of conjunctival mucin $5 \mathrm{AC}$ and aquaporin 5 in response to acute dry eye stress. PLoS ONE. 2017; 12: e0187188.

[3] Yu L, Bhattacharya D, Wang Z, Wang M. Topical administration of ambroxol eye drops augments tear secretion in rabbits. Graefe'S Archive for Clinical and Experimental Ophthalmology. 2021; 259: 1529-1538.

[4] Cazan D, Klimek L, Sperl A, Plomer M, Kölsch S. Safety of ambroxol in the treatment of airway diseases in adult patients. Expert Opinion on Drug Safety. 2018; 17: 1211-1224.

[5] Germouty J, Jirou-Najou JL. Clinical efficacy of ambroxol in the treatment of bronchial stasis. Clinical trial in 120 patients at two different doses. Respiration; International Review of Thoracic Diseases. 1987; 51: 37-41.

[6] Beeh KM, Beier J, Esperester A, Paul LD. Antiinflammatory properties of ambroxol. European Journal of Medical Research. 2008; 13: 557-562.

[7] Gaida W, Klinder K, Arndt K, Weiser T. Ambroxol, a Nav1.8 preferring $\mathrm{Na}(+)$ channel blocker, effectively suppresses pain symptoms in animal models of chronic, neuropathic and inflammatory pain. Neuropharmacology. 2005; 49: 1220-1227.

[8] Schrader S, Mircheff AK, Geerling G. Animal models of dry eye. Developments in Ophthalmology. 2008; 41: 298-312.

[9] Honkanen RA, Huang L, Xie G, Rigas B. Phosphosulindac is efficacious in an improved concanavalin a-based rabbit model of chronic dry eye disease. Translational Research. 2018; 198: $58-72$.

[10] Demetriades AM, Leyngold IM, D’Anna S, Eghrari AO, Emmert DG, Grant MP, et al. Intraglandular injection of botulinum toxin a reduces tear production in rabbits. Ophthalmic Plastic and Reconstructive Surgery. 2013; 29: 21-24.

[11] Bhattacharya D, Ning Y, Zhao F, Stevenson W, Chen R, Zhang $\mathrm{J}$, et al. Tear Production after Bilateral Main Lacrimal Gland Resection in Rabbits. Investigative Ophthalmology \& Visual Science. 2015; 56: 7774-7783.

[12] Ning Y, Bhattacharya D, Jones RE, Zhao F, Chen R, Zhang $\mathrm{J}$, et al. Evaluating the Functionality of Conjunctiva Using a 
Rabbit Dry Eye Model. Journal of Ophthalmology. 2016; 2016 : 3964642.

[13] Honkanen R, Huang W, Huang L, Kaplowitz K, Weissbart S, Rigas B. A New Rabbit Model of Chronic Dry Eye Disease Induced by Complete Surgical Dacryoadenectomy. Current Eye Research. 2019; 44: 863-872.

[14] Stapleton F, Alves M, Bunya VY, Jalbert I, Lekhanont K, Malet $\mathrm{F}$, et al. TFOS DEWS II Epidemiology Report. The Ocular Surface. 2017; 15: 334-365.

[15] Bron AJ, de Paiva CS, Chauhan SK, et al. TFOS DEWS II pathophysiology report. Ocular Surface. 2017; 15: 438-510.

[16] Schechter JE, Warren DW, Mircheff AK. A lacrimal gland is a lacrimal gland, but rodent's and rabbit's are not human. The Ocular Surface. 2010; 8: 111-134.

[17] Gipson IK. Goblet cells of the conjunctiva: a review of recent findings. Progress in Retinal and Eye Research. 2016; 54: 4963.

[18] Gipson IK, Argüeso P. Role of mucins in the function of the corneal and conjunctival epithelia. International Review of Cytology. 2003; 231: 1-49.

[19] Blalock TD, Spurr-Michaud SJ, Tisdale AS, Gipson IK. Release of membrane-associated mucins from ocular surface epithelia. Investigative Ophthalmology \& Visual Science. 2008; 49: 1864-1871.

[20] Argueso P, Balaram M, Spurr-Michaud S, et al. Decreased levels of the goblet cell mucin MUC5AC in tears of patients with Sjogren syndrome. Investigative Ophthalmology \& Visual Science. 2002; 43: 1004-1011.

[21] Wang L, Yu L, Li N, Wang Y, Yang M, Peng Y, et al. Bromhexine elevates REP2 expression to stimulate secretion from human primary conjunctiva fornix epithelial cells. FEBS Letters. 2020; 594: $153-160$.
[22] de Oliveira S, Reyes-Aldasoro CC, Candel S, Renshaw SA Mulero V, Calado A. Cxcl8 (IL-8) mediates neutrophil recruitment and behavior in the zebrafish inflammatory response. Journal of Immunology. 2013; 190: 4349-4359.

[23] Yamaya M, Nishimura H, Nadine LK, Ota C, Kubo H, Nagatomi R. Ambroxol inhibits rhinovirus infection in primary cultures of human tracheal epithelial cells. Archives of Pharmacal Research. 2014; 37: 520-529.

[24] Gao J, Morgan G, Tieu D, et al. ICAM-1 expression predisposes ocular tissues to immune-based inflammation in dry eye patients and Sjogrens syndrome-like MRL/lpr mice. Experimental Eye Research. 2004; 78: 823-835.

[25] Tauber J, Karpecki P, Latkany R, Luchs J, Martel J, Sall K, et al. Lifitegrast Ophthalmic Solution 5.0\% versus Placebo for Treatment of Dry Eye Disease: Results of the Randomized Phase III OPUS-2 Study. Ophthalmology. 2015; 122: 2423-2431.

[26] Varelogianni G, Hussain R, Strid H, Oliynyk I, Roomans GM, Johannesson M. The effect of ambroxol on chloride transport, CFTR and $\mathrm{ENaC}$ in cystic fibrosis airway epithelial cells. Cell Biology International. 2014; 37: 1149-1156.

[27] Stevenson W, Pugazhendhi S, Wang M. Is the main lacrimal gland indispensable? Contributions of the corneal and conjunctival epithelia. Survey of Ophthalmology. 2016; 61: 616-627.

[28] Terakado K, Yogo T, Kohara Y, Soeta S, Nezu Y, Harada Y, et $a l$. Conjunctival expression of the $\mathrm{P} 2 \mathrm{Y} 2$ receptor and the effects of $3 \%$ diquafosol ophthalmic solution in dogs. The Veterinary Journal. 2014; 202: 48-52.

[29] Urashima H, Takeji Y, Okamoto T, Fujisawa S, Shinohara H. Rebamipide Increases Mucin-Like Substance Contents and Periodic Acid Schiff Reagent-Positive Cells Density in Normal Rabbits. Journal of Ocular Pharmacology and Therapeutics. 2012; 28: 264-270. 